\title{
Case against routine use of glyceryl trinitrate before coronary angiography ${ }^{1,2}$
}

\author{
ROBERT L. FELDMAN, CARL J. PEPINE, R. CHARLES CURRY, JR., AND \\ C. RICHARD CONTI \\ From the Division of Cardiovascular Medicine, University of Florida, Gainesville, Florida, USA
}

SUMMARY Changes in coronary artery tone are often neglected in the angiographic assessment of patients with chest pain. To determine the contributions of dynamic coronary artery diameter changes to the degree of coronary narrowing, 47 consecutive chest pain patients were studied without premedication by analysing selective coronary angiograms before and after glyceryl trinitrate. Proximal ؟ coronary diameters were measured in superimposed frames with callipers. Lumen diameter increased after glyceryl trinitrate $15 \pm 2$ per cent in the left main, $18 \pm 2$ per cent in the left anterior descending, $16 \pm 2$ per cent in the circumflex, and $17 \pm 2$ per cent in the right coronary arteries (all $P<0.01$ ). One hundred and nineteen coronary artery narrowings of $<90$ per cent were measured (narrowings $>90$ per cent were excluded because of measurement inaccuracy). Of 81 narrowings $<50$ per cent, 24 became $\geq 50$ per cent because of dilatation of the adjacent region. However, the diameter of the narrowed segment also changed significantly after glyceryl trinitrate, increasing in 28 and decreasing in 3. Of the 28 obstructions which dilated after glyceryl trinitrate, 4 were clinical and angiographic examples of coronary arterial spasm. In 4 other coronary arteries with only outline irregularities before glyceryl trinitrate a localised narrowing (25 to $50 \%$ ) was seen after glyceryl trinitrate as the proximal artery dilated. We concluded that the routine use of glyceryl trinitrate before coronary angiography alters the measured degree of coronary artery narrowing and obscures the angiographic interpretation.

When nitrates are given before coronary angiography, the coronary artery image often appears enhanced. For this reason, sublingual glyceryl trinitrate has been used routinely before coronary angiography in many laboratories (Baltaxe et al., 1973; Bennett, 1976; Chahine and Luchi, 1976). Some have suggested that the angiographically observed dilatory response of normal coronary arteries differs quantitatively from diseased arteries (Gensini et al., 1971). This raises questions relating to the interpretation of nitrate-induced dilatation of diseased and non-diseased sections of the coronary artery as seen angiographically. Different dilator responses in these areas could distort the angiographic estimate of the degree of coronary artery

\footnotetext{
${ }^{1} \mathrm{~A}$ preliminary report of this work was presented at the 49th Scientific Sessions of the American Heart Association, Miami, Florida-15-18 November 1976.

${ }^{2}$ This work was supported in part by an HEW Grant and an NIH Grant.
}

Received for publication 30 November 1977 narrowing. Additionally, recent interest in the role of coronary arterial spasm in the pathogenesis of myocardial ischaemia suggests that spasm may be more common than generally recognised (Bennett, 1976; Chahine and Luchi, 1976; Weiner et al., 1976). Routine use of glyceryl trinitrate before or at the start of an angiographic procedure could have a profound influence on the angiographic interpretation in this setting. Accordingly, the purpose of this study was to assess the effect of glyceryl trinitrate on the angiographic interpretation of coronary artery narrowing.

\section{Methods}

Studies were made on 47 consecutive, unpremedicated patients undergoing preoperative evaluation for chest pain. Patients did not receive nitrates for at least 2 hours before the study.

The coronary angiographic procedure was conducted according to standard techniques. Aortic pressure and electrocardiographic leads were constantly monitored. 


\section{ANGIOGRAPHIC AND MEASUREMENT}

TECHNIQUES

Selective coronary injections of Renografin 76 (TM) were made by hand in multiple projections before (before GTN) and after sublingual glyceryl trinitrate (GTN), $0.4 \mathrm{mg}$. High quality coronary cineangiograms at $60 \mathrm{frames} / \mathrm{s}$ were obtained using $4 \frac{1}{2}$ or 6 in image intensification and a $100 \mathrm{~mm}$ overframing lens with Ilford Type CF film. The resolution in our two catheterisation laboratories is 2.8 and 3.0 line pairs $/ \mathrm{mm}$. Patient position was the same, before and after GTN. This was accomplished by measuring the cardiac apex to table top distance (RAO) or cradle angle (LAO). Similarly, relations between focal spot, patient, and image tube height were kept constant. Because of possible transient vasodilatory effects of Renografin 76 (TM), a minimum of 5 minutes was allowed between coronary injections (Hattenhauer and Neill, 1975).

Selected cineframes from before and after GTN arteriograms were matched for the same part of the cardiac cycle. End-diastolic frames were chosen so that maximal coronary diameters could be analysed. Cineframes were projected on a viewing screen (Tagarno, Model 3500). Coronary artery size was determined by measuring the diameters of the 4 major proximal coronary arteries and coronary narrowings with calipers to the nearest $0.5 \mathrm{~mm}$. Proximal coronary arteries were measured at branch points and narrowings at their minimal diameter. Diameter measurements from control angiograms (before GTN) were then compared with diameter measurements of the same portion of the coronary artery on the GTN angiogram. We found that the projected diameter of a coronary narrowing less than $1.0 \mathrm{~mm}$ could not be measured accurately. Therefore, those narrowings (generally $90 \%$ or greater) were not included in this report. The degree of narrowing was expressed as a percentage of coronary narrowing. The latter was calculated from the ratio of the diameter at the narrowing to the diameter of the coronary artery immediately proximal to the narrowing $\times 100$.

REPRODUCIBILITY AND SPONTANEOUS VARIATION OF CORONARY ARTERY MEASUREMENTS

The ability to reproduce measured projected image diameters was evaluated by the following studies. Multiple measurements of projected diameters of intracardiac or intra-aortic catheters, proximal coronary arteries, and coronary artery narrowings were made from cineangiographic sequences. For each of these, 25 to 35 individual measurements were made from sequential frames of five to six sequences. Coefficients of variation for each measurement were calculated. Variation of measure- ments of the same projected diameter (i.e. catheter tip) served as an internal control.

Additionally, in 5 other patients possible spontaneous changes in coronary artery diameter were evaluated by repeating the coronary angiography studies 30 to 60 minutes after the control series of angiograms and before administration of GTN. Eighteen proximal coronary arteries were evaluated in this manner.

Means and standard errors of the mean and coefficients of variation (Snedecor and Cochran, 1973) were calculated for the measured diameters and their ratios. Student's ' $t$ ' test was used to determine the statistical significance of the difference between the mean values of before GTN and GTN observations. A P value $<0.05$ was considered statistically significant.

\section{Results}

MEASUREMENT REPRODUCIBILITY AND SPONTANEOUS VARIATION IN CORONARY ARTERY DIAMETER

The mean value for measurements of the projected diameter of aortic catheters in 5 patients ranged from 4.5 to $8.0 \mathrm{~mm}$ and their respective coefficients of variation ranged from 0 to 5 per cent. The projected mean diameter of 6 coronary arteries ranged from 8 to $12.5 \mathrm{~mm}$ as their respective coefficients of variation ranged from 0 to 3 per cent. The projected mean diameters of 5 coronary narrowings ranged from 3 to $5 \mathrm{~mm}$ as their respective coefficients of variation ranged from 0 to 7 per cent. Coronary diameter measurements repeated after 30 to 60 minutes without GTN showed that one coronary artery decreased in size, 16 remained unchanged, and 1 enlarged. The net change was an increase of $2 \pm 3$ per cent (mean \pm standard error); $P>0.05$.

\section{EFFECT OF GLYCERYL TRINITRATE ON CORONARY ARTERY MEASUREMENTS}

\section{Effects on proximal coronary arteries}

One-hundred and sixty-nine proximal coronary artery diameters were measured before and after GTN. These diameters increased as follows: left main $15 \pm 2$ per cent, anterior descending branch $18 \pm 2$ per cent, circumflex branch $16 \pm 2$ per cent, and right coronary artery $17 \pm 2$ per cent (all $P<0.01)$. There was no significant difference in the degree of changes induced by GTN between the 4 arteries studied. The dilator effect was not related to the size of the artery or the presence or absence of coronary artery disease. There was pronounced individual variability both in magnitude and direction of the changes. Exclud- 

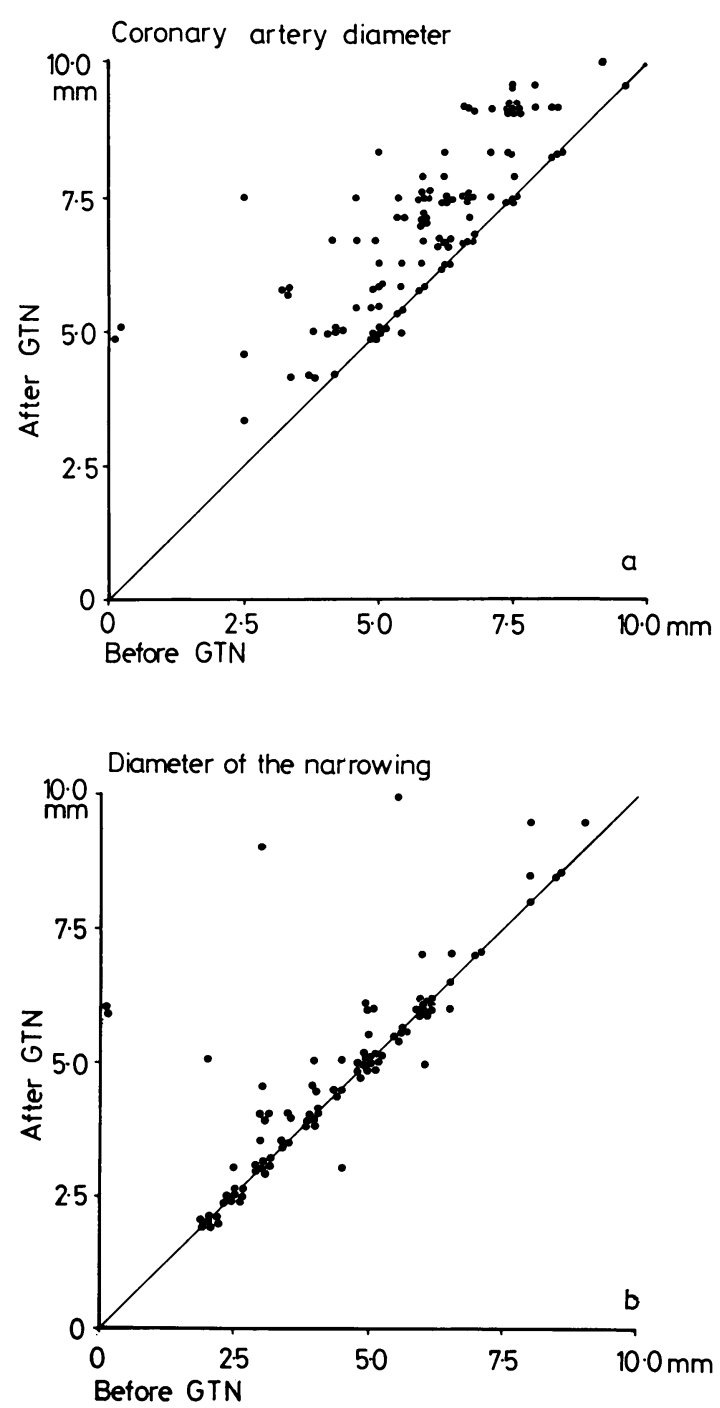

Fig. $1 \mathrm{a}$ and $\mathrm{b}$ Effect of GTN on the diameter of the projected proximal coronary arteries (a) and diameter of the coronary narrowing (b). Measurements from the before GTN angiogram (horizontal axis) and after GTN (vertical axis) are compared. The solid line represents the line of identity. Dilatation after GTN is represented by points above the identity line. The majority of the major proximal vessels dilate (panel a) while the majority of narrowings do not change diameter (panel $\mathrm{b}$ ).

ing instances of definite coronary arterial spasm, the diameter changes ranged from a 6 per cent decrease to a 57 per cent increase. The diameter of one proximal artery decreased, 24 arteries remained unchanged, and 144 arteries increased. These coronary responses are summarised in Fig. 1a.

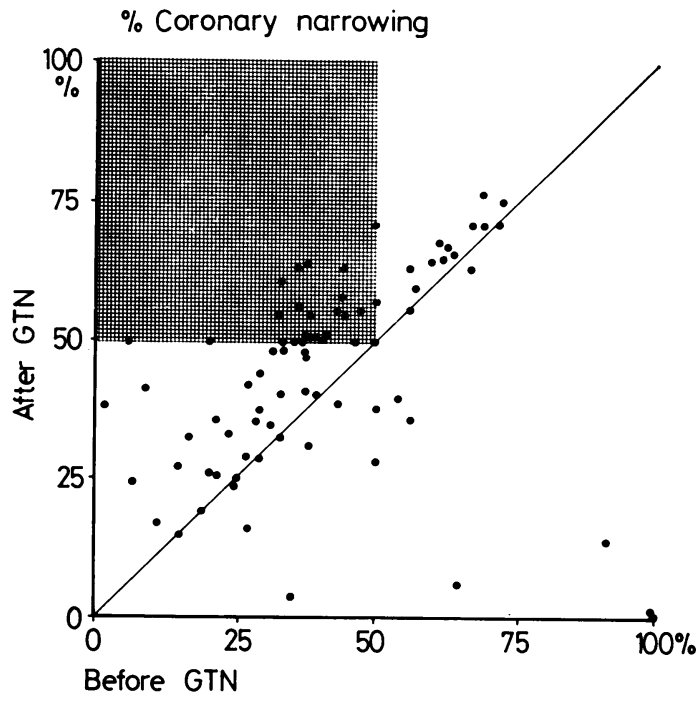

Fig. 2 Effect of GTN on percentage coronary narrowing. The percentage coronary narrowing, before GTN (horizontal) is compared with a percentage narrowing after GTN (vertical). In most cases, the degree of narrowing increased after GTN. Points lying below the line of identity may represent coronary arterial spasm. The subgroup included in the hatched area (upper left quadrant) represents narrowings measuring $<50$ per cent before GTN and > 50 per cent after GTN.

Effect of glyceryl trinitrate on coronary artery narrowings

Changes in diameter of the 119 coronary narrowings measured are summarised in Fig. 1b. Three narrowings decreased in diameter, 88 remained unchanged, and 28 increased.

Effect of glyceryl trinitrate on percentage narrowing The influence of GTN on the diameter of the coronary narrowings relative to the diameter of the proximal arteries is shown in Fig. 2. Overall, glyceryl trinitrate produced a small ( $8 \%$ mean) but significant $(P<0.01)$ increase in percentage narrowing. Variability was apparent as the percentage narrowing of 15 obstructions decreased, 17 remained unchanged, and 87 increased. Changes in the percentage narrowing were observed for all geometric types and degrees of obstruction. The subgroup of narrowings measuring less than 50 per cent diameter reduction before GTN were of particular interest. Eighty-one narrowings were in this subset. Of these narrowings, 24 showed an apparent increase to 50 per cent narrowing or greater after GTN (stippled area of Fig. 2). The increase in the degree of narrowing of the obstruction within this subset ranged from 4 to 30 per cent. 
Effect of glyceryl trinitrate on haemodynamic measurements

After the administration of sublingual GTN mean aortic blood pressure decreased $10 \mathrm{mmHg}$ (average $(P<0.05)$, range 4 to $15 \mathrm{mmHg}$. Heart rate increased 6 beats $/ \mathrm{min}$ (average $P<0.05$ ), range 0 to 11 beats $/ \mathrm{min}$.

\section{Discussion}

Although glyceryl trinitrate is routinely administered before coronary angiography in many laboratories (Baltaxe et al., 1973; Bennett, 1976; Chahine and
Luchi, 1976), its precise effects on coronary arteries and arterial narrowings is unclear. Furthermore, its influence on the interpretation of coronary angiograms has not been reported previously.

Our results indicate that a small increase in the degree of coronary narrowing $(8 \%$ mean) and the proximal coronary diameter (17\% mean) occurs after glyceryl trinitrate. Though statistically significant, these changes were not predictable in the individual patient. Gensini and co-workers (1971) using isosorbide dinitrate made similar observations. In our patients, the change in degree of coronary narrowing after sublingual glyceryl trinitrate was

(a)
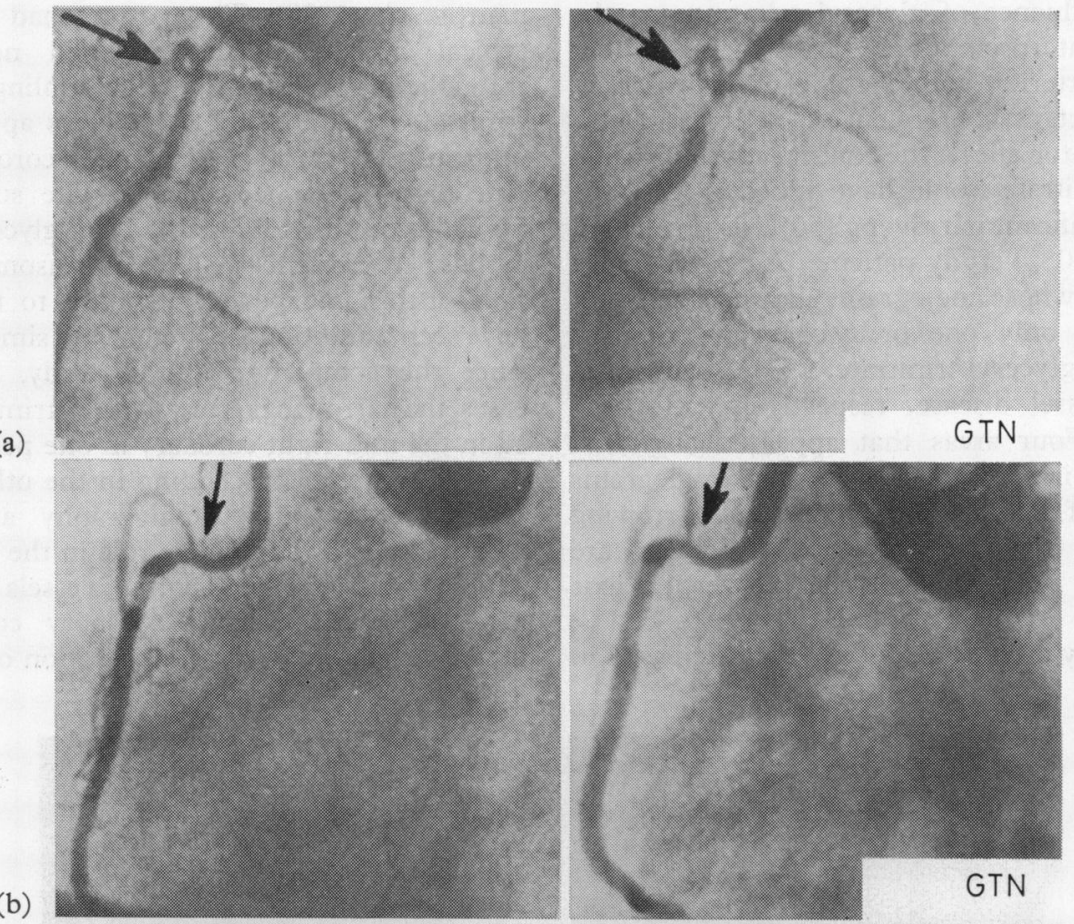

(c)
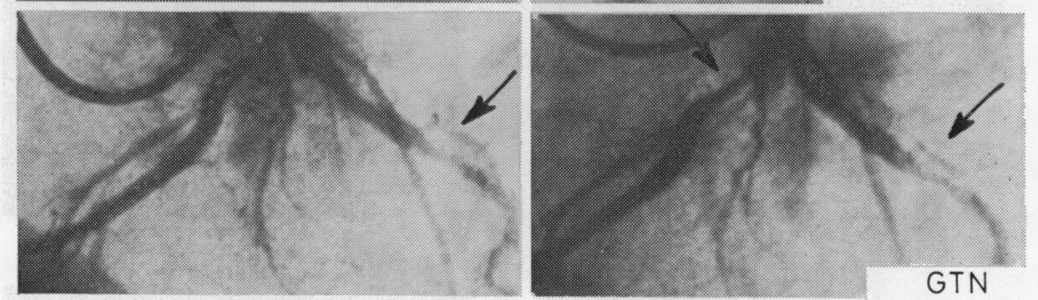

Fig. 3a, b, and c Examples illustrating the distortion glyceryl trinitrate can produce in the interpretation of the degree of coronary narrowing: before GTN angiograms (left) are compared with after GTN angiograms (right). Two patients with $<50$ per cent narrowing of the proximal right coronary (LAO projection), panel $\mathrm{a}$ and $\mathrm{b}$, and another with two $<50$ per cent narrowings of the left coronary (LAO projection), panel c, are shown. In each case after GTN, disproportionate dilatation of the adjacent vessel relative to the narrowed segment (arrow) caused an apparent increase in the degree of narrowing. 
largely the result of dilatation of the proximal coronary artery relative to the narrowing. An important clinical implication relative to this observation is that approximately one-third of the narrowings measuring $<50$ per cent before glyceryl trinitrate, measured $\geqslant 50$ per cent after glyceryl trinitrate (Fig. 2, stippled area). While few cardiologists would consider these narrowings clinically significant before glyceryl trinitrate, many would interpret them as significant obstructions after glyceryl trinitrate. Several such narrowings measured less than 25 per cent before the drug but measured $\geqslant 50$ per cent after it. Examples of this type of change are illustrated in Fig. 3. Consideration of the influence of glyceryl trinitrate on the angiographic interpretation, in terms of the number of coronary arteries with significant obstruction, also has important consequences. Because of this differential dilator effect, the routine administration of glyceryl trinitrate would have added at least one additional significant narrowing (50\% or greater) in 14 of the $47(30 \%)$ study patients. Additionally, of the 16 patients whose angiograms showed significant obstruction of only one or two major coronary arteries before glyceryl trinitrate, $5(31 \%)$ advanced to 2- or 3-vessel disease, respectively, after administration. Four areas that appeared as trivial lumen outline irregularities on control angiograms became areas of definite 25 to 50 per cent narrowing after glyceryl trinitrate (these narrowings are represented by points lying along the vertical axis of Fig. 2).

The coronary artery narrowings that increased in size also merit comment considering recent interest in the role of coronary artery spasm in the pathophysiology of myocardial ischaemia. In this regard, 28 of 119 narrowings enlarged after glyceryl trinitrate (Fig. 1b). Of these, at least 4 were considered to be examples of coronary spasm. However, many of the other 24 narrowings may represent combinations of atherosclerosis (fixed obstruction) and spasm (reversible obstruction). Alternatively, they could also represent localised atherosclerotic-type changes that do not involve the entire circumference of a vessel.

One patient developed an unexpected response after glyceryl trinitrate as illustrated in Fig. 4 (Feldman et al., 1978). The patient had a history of typical angina pectoris but had no symptoms throughout the study. After sublingual glyceryl trinitrate, new areas of narrowings appeared in the mid-portion of his large right coronary artery. The narrowings persisted, despite sublingual administration of additional $0.4 \mathrm{mg}$ glyceryl trinitrate tablets. At present, we have no reasonable explanation for this unexpected response to the drug but have seen 2 additional patients with similar responses since the completion of this study. In the latter cases, the narrowings after glyceryl trinitrate appeared in the mid-right coronary in one patient and the mid-left anterior descending in the other patient.

Selective coronary angiography after glyceryl trinitrate has an adjunctive role in the evaluation of patients with chest pain. Often, vessels distal to high grade obstructions and coronary collaterals are better visualised after administration of nitrates. In

Fig. 4 Example of an unexpected response observed after GTN. Note proximal and distal vessel dilatation after GTN (right) but areas of narrowing appear which were not apparent before GTN (left). Repeated angiograms after additional $0.4 \mathrm{mg}$ GTN tablets to a total dose of $1.2 \mathrm{mg}$ showed no change. (Reproduced by permission of American Journal of Cardiology.) 
addition, glyceryl trinitrate can help to distinguish fixed narrowings from those with superimposed spasm (Gensini, 1975; Chahine and Luchi, 1976). In patients with coronary narrowings on routine angiograms, another set of arteriograms after glyceryl trinitrate seems appropriate in order to have an improved assessment of possible dynamic changes (Chahine and Luchi, 1976). However, since part of the risk of coronary arteriography is related to the length of the procedure, sound judgement dictates the type of study for each individual patient.

The observations reported here are valid only if the methods are reproducible and accurate and the coronary artery diameter is stable throughout the catheterisation procedure. Reproducibility and accuracy can be accomplished by controlling patient and equipment position, using high quality cineangiographic techniques, and making calibrated measurement with a low coefficient of variation. In our unpremedicated patients, there was no significant change in coronary artery diameter over a 30 to 45 minute period provided the patient did not experience spontaneous angina. During spontaneous chest pain, however, there may be associated changes in coronary size, as we observed and as others have suggested (Weiner et al., 1976).

The methods used include other pertinent limitations. First, coronary artery diameter measurements were made only at specific coronary artery sites; since measurements were not made over the entire coronary artery, we cannot exclude possible diameter changes occurring in other areas. Second, though high quality cineangiography was used, the borders of high degree narrowings (usually $\geqslant 90 \%$ and measuring $\geqslant 1 \mathrm{~mm}$ ) were at times indistinct; therefore, measurements from these areas were excluded from this report. Third, since measurements were not made in a blind manner, some degree of subjectivity could be associated with the calliper measurements.

In conclusion, these observations indicate that administration of glyceryl trinitrate before coronary angiography can alter the interpretation of the coronary angiogram. This alteration consists of changes in percentage narrowing of the vessel. In most cases, disproportionate dilatation of the vessel adjacent to a narrowing is responsible for these changes. In some patients, however, distinct changes also occur at the narrowing. Some of these changes may represent coronary spasm that could have been obscured if glyceryl trinitrate had been given before the angiogram.

\section{References}

Baltaxe, H. A., Amplatz, K., and Levin, D. C. (1973). Coronary Angiography. Charles C. Thomas, Springfield, Illinois.

Bennett, K. R. (1976). Coronary artery spasm; the effect of cardiovascular laboratory premedication practice. Catheterization and Cardiovascular Diagnosis, 2, 321-327.

Chahine, R. A., and Luchi, R. J. (1976). Coronary arterial spasm: culprit or bystander? American fournal of Cardiology, 37, 936-937.

Feldman, R. L., Pepine, C. J., and Conti, C. R. (1978). Unusual isometer coronary arterial reponses occurring after nitroglycerin. American fournal of Cardiology, 42, (in press).

Gensini, G. G. (1975). Coronary Arteriography. Futura, Mount Kisco, N.Y.

Gensini, G. G., Kelly, A. E., Da Costa, B. C. B., and Huntington, P. P. (1971). Quantitative angiography: the measurement of coronary vasomobility in the intact animal and man. Chest, 60, 522-530.

Hattenhauer, M., and Neill, W. A. (1975). Effect of cold air inhalation on angina pectoris and myocardial oxygen supply. Circulation, 51, 1053-1058.

Snedecor, G. W., and Cochran, W. G. (1973). Statistical Methods, 6th ed. The Iowa State University Press, Ames, Iowa.

Weiner, L., Kasparian, H., Duca, P. R., Walinsky, P., Gottlieb, R. S., Hanckel, F., and Brest, A. (1976). Spectrum of coronary arterial spasm. Clinical, angiographic and myocardial metabolic experience in 29 cases. American fournal of Cardiology, 33, 945-955.

Requests for reprints to Dr C. Richard Conti, Division of Cardiovascular Medicine, University of Florida, Box J-277, J. Hillis Miller Health Center, Gainesville, Florida 32610, USA. 